\title{
Relation between glycaemic control, hyperinsulinaemia and plasma concentrations of soluble adhesion molecules in patients with impaired glucose tolerance or Type II diabetes
}

\author{
M. Blüher ${ }^{1}$, R. Unger ${ }^{2}$, F. Rassoul ${ }^{2}$, V. Richter $^{2}$, R.Paschke ${ }^{1}$ \\ ${ }^{1}$ III Medical Department, University of Leipzig, Leipzig, Germany \\ ${ }^{2}$ Institute of Clinical Chemistry and Pathobiochemistry, University of Leipzig, Leipzig, Germany
}

\section{Abstract}

Aims/hypothesis. Increased plasma concentrations of circulating adhesion molecules in patients with Type II (non-insulin-dependent) diabetes mellitus could be associated with the increased cardiovascular risk in these patients. However, it is controversial whether increased adhesion molecule plasma concentrations are primarily related to hyperglycaemia or to hyperinsulinaemia.

Methods. We evaluated the plasma concentrations of soluble E-selectin, intracellular adhesion molecule-1 (ICAM-1), and vascular cell adhesion molecule-1 (VCAM-1) at baseline and during euglycaemic hyperinsulinaemic clamp in three different groups without additional cardiovascular risk factors: group A (control group), 28 healthy volunteers with normal glucose tolerance; group B, 24 subjects with fasting hyperinsulinaemia, normal fasting glucose but impaired glucose tolerance; group C, 32 patients with Type II diabetes, fasting hyperinsulinaemia and chronic hyperglycaemia.

Results. Plasma soluble E-selectin, ICAM-1, and VCAM-1 concentrations were higher $(p<0.05)$ in patients with Type II diabetes (group C) compared with the other groups. The adhesion molecule concentrations correlate with the fasting plasma glucose $(r=0.59, p<0.001)$, the 2-h OGTT plasma glucose $(r=0.70, p<0.01)$, and the $\mathrm{HbA}_{1 \mathrm{c}}$ value $(r=0.61$, $p<0.05)$. The E-selectin but not the ICAM-1 and VCAM-1 plasma concentrations correlated with the fasting insulin concentrations $(r=0.62, p<0.05)$ or the whole body glucose uptake $(r=0.59, p<0.05)$ in the clamp. The hyperinsulinaemia during the euglycaemic hyperinsulinaemic clamp had no significant effect on the plasma concentrations of E-selectin, ICAM-1, and VCAM-1 in all three groups.

Conclusion/interpretation. Our results suggest that increased E-selectin concentrations are related to hyperglycaemia, hyperinsulinaemia and insulin resistance, whereas increased ICAM-1 and VCAM- 1 plasma concentrations in patients with Type II diabetes are rather related to hyperglycaemia than to hyperinsulinaemia or insulin resistance. [Diabetologia (2002) 45: 210-216]

Keywords E-selectin, ICAM-1, VCAM-1, insulin resistance, glycaemic control.
Received: 13 August 2001 and in revised form: 1 October 2001

Corresponding author: R. Paschke, III Medical Department, University of Leipzig, Philipp-Rosenthal-Straße 27, Leipzig, Germany, e-mail: pasr@medizin.uni-leipzig.de

Abbreviations: AM, Adhesion molecules; ICAM-1, intracellular adhesion molecule-1; VCAM-1, vascular cell adhesion molecule-1; ADA, American Diabetes Association; SBP, systolic blood pressure; DBP, diastolic blood pressure
Patients with Type II (non-insulin-dependent) diabetes mellitus have an increased risk for premature atherosclerosis $[1,2]$. Increased concentrations of plasma adhesion molecules (AM) have been detected in patients with developing atherosclerosis [3] and in patients with Type I (insulin-dependent) diabetes mellitus [4, 5] and Type II diabetes [6-9]. Moreover, increased plasma concentrations of soluble forms of some of these adhesion molecules have been suggested as markers of early atherosclerosis $[10,11]$. These 
adhesion molecules include intercellular cell adhesion molecules-1 (ICAM-1), vascular cell adhesion molecules-1 (VCAM-1), and E-selectin, a specific product of endothelial cells [12]. However, it is still controversial as to whether the increased plasma concentrations of AM are primarily the result of hyperglycaemia $[7,8]$ or the hyperinsulinaemia in the insulin resistant state $[3,14]$. Although it was recently shown that short-term hyperinsulinaemia during a $6 \mathrm{~h}$ euglycaemic hyperinsulinaemic clamp did not increase plasma AM concentrations [15], the effect of chronic hyperinsulinaemia on plasma AM concentrations requires further investigation, because identification of the primary factors inducing increased AM concentrations in patients with diabetes might have further implications in the primary prevention of cardiovascular disease in these patients.

We therefore evaluated whether increased plasma concentrations of ICAM-1, VCAM-1, and E-selectin are primarily associated with either hyperglycaemia or hyperinsulinaemia and insulin resistance. To avoid confounding factors which could affect plasma AM concentrations, we selected subjects without clinical evidence of vascular lesions, and without other cardiovascular risk factors like hyperlipidaemia, hypertension or smoking. To evaluate the effect of chronic hyperinsulinaemia on AM concentrations, we compared plasma AM concentrations in subjects with IGT, extreme insulin resistance and fasting hyperinsulinaemia, but still normal fasting plasma glucose and $\mathrm{HbA}_{1 \mathrm{c}}$ values, to the concentrations in normal control subjects. For the evaluation of the effects of combined chronic hyperglycaemia and hyperinsulinaemia, AM plasma concentrations were measured in patients with newly diagnosed Type II diabetes who had evidence for accompanying chronic hyperglycaemia. Moreover, we investigated the short time effects of hyperinsulinaemia on ICAM-1, VCAM-1, and E-selectin plasma concentrations with euglycaemic hyperinsulinaemic clamps in all study groups. Based on these groups, we tested the hypothesis that increased plasma concentrations of soluble adhesion molecules are primarily related to chronic hyperglycaemia rather than to insulin resistance.

\section{Subjects and methods}

Subjects. A total of 84 Caucasian men and women (mean age $39.6 \pm 5.4$ years) were selected from 469 subjects, which were screened for impaired glucose tolerance by a $75 \mathrm{~g}$ OGTT according to the American Diabetes Association (ADA) criteria [16] with the aim of obtaining three different groups matched for age, sex and BMI.

Group A included subjects with normal glucose tolerance, defined by fasting plasma glucose of less than $6.0 \mathrm{mmol} / \mathrm{l}[16]$ and $120 \mathrm{~min}$ plasma glucose after a $75 \mathrm{~g}$ oral glucose load of less than $7.8 \mathrm{mmol} / \mathrm{l}$, without hyperinsulinaemia and without a family history of Type II diabetes or diabetes during pregnancy ( $n=28,10$ men, 18 women).

Group B included subjects with impaired glucose tolerance, defined by a fasting plasma glucose of less than $6.0 \mathrm{mmol} / \mathrm{l}$ and a 120 min plasma glucose after $75 \mathrm{~g}$ oral glucose load of less than $11.1 \mathrm{mmol} / \mathrm{l}$ [16], fasting hyperinsulinaemia $(n=24$, 10 men, 14 women).

Group C included patients with newly diagnosed Type II diabetes, defined by a fasting plasma glucose greater than $6 \mathrm{mmol} / \mathrm{l}$ and/or a $120 \mathrm{~min}$ OGTT glucose greater than $11.1 \mathrm{mmol} / \mathrm{l}$, fasting hyperinsulinaemia $(n=32,15 \mathrm{men}, 17$ women).

All subjects were chosen according to the following inclusion criteria:

- no history of any metabolic disorder (except from the Type II diabetic patients in group $\mathrm{C}$ ):

- glutamic acid decarboxylase (GAD) measured by an RIA (BRAHMS, Berlin, Germany) and/or islet cell antibody determined by an immunohistochemical method undetectable;

- no dyslipidemia (LDL-cholesterol > $3.9 \mathrm{mmol} / \mathrm{l}$ );

- no medical history of hypertension, (i.e. systolic blood pressure $(\mathrm{SBP})<160 \mathrm{mmHg}$ and diastolic blood pressure (DBP) $<95 \mathrm{mmHg}$, using the criteria of the European Group for the Study of Insulin Resistance ESIR [17]);

- no clinical evidence for either cardiovascular or peripheral artery disease (no history of an acute myocardial infarction, no ECG abnormalities, no history of bypass surgery or coronary angioplasty, no clinical symptoms of typical angina, dyspnoe or claudicatio intermittens);

- no thyroid dysfunction;

- no concomitant medication;

- no regular alcohol or drug consumption and no smoking; and

- no pregnancy.

Systolic and diastolic blood pressure were not significantly different between the groups. In addition to the absence of any additional cardiovascular risk factors except Type II diabetes there was no evidence of diabetic retinopathy or nephropathy in these patients. The study was approved by the ethics committee of the University of Leipzig. All subjects gave written informed consent before taking part in the study.

Oral glucose tolerance test. The OGTT was done less than 1 week before the euglycaemic hyperinsulinaemic clamps and the measurements of the AM according to the criteria of the ADA [16]. Three days before the OGTT the patients documented a high carbohydrate diet. The OGTT was done after an overnight fast with a $75 \mathrm{~g}$ standardised glucose solution (Glucodex solution 75 g, Merieux, North York, Canada). Venous blood samples were taken at 0, 60, 90, and $120 \mathrm{~min}$ for measurements of plasma glucose, insulin and C-peptide concentrations.

Euglycaemic-hyperinsulinaemic-glucose-clamp. Insulin sensitivity was assessed with the euglycaemic, hyperinsulinaemic clamp method [18]. Subjects with a whole body glucose uptake of less than $40 \mu \mathrm{mol} \cdot \mathrm{kg}^{-1} \cdot \mathrm{min}^{-1}$ were defined as insulin resistant and subjects with a whole body glucose uptake greater than $50 \mu \mathrm{mol} \cdot \mathrm{kg}^{-1} \cdot \mathrm{min}^{-1}$ were defined as insulin sensitive according to criteria described previously [19]. There were no subjects with a whole body glucose uptake between 40 and $50 \mu \mathrm{mol} \cdot \mathrm{kg}^{-1} \cdot \mathrm{min}^{-1}$. After an overnight fast and resting for $30 \mathrm{~min}$ in a supine position, intravenous catheters were inserted into antecubital veins in both arms of the participants. One was used for the infusion of insulin and glucose, the other was 
Table 1. Clinical and biochemical characteristics (means \pm SEM) of the probands

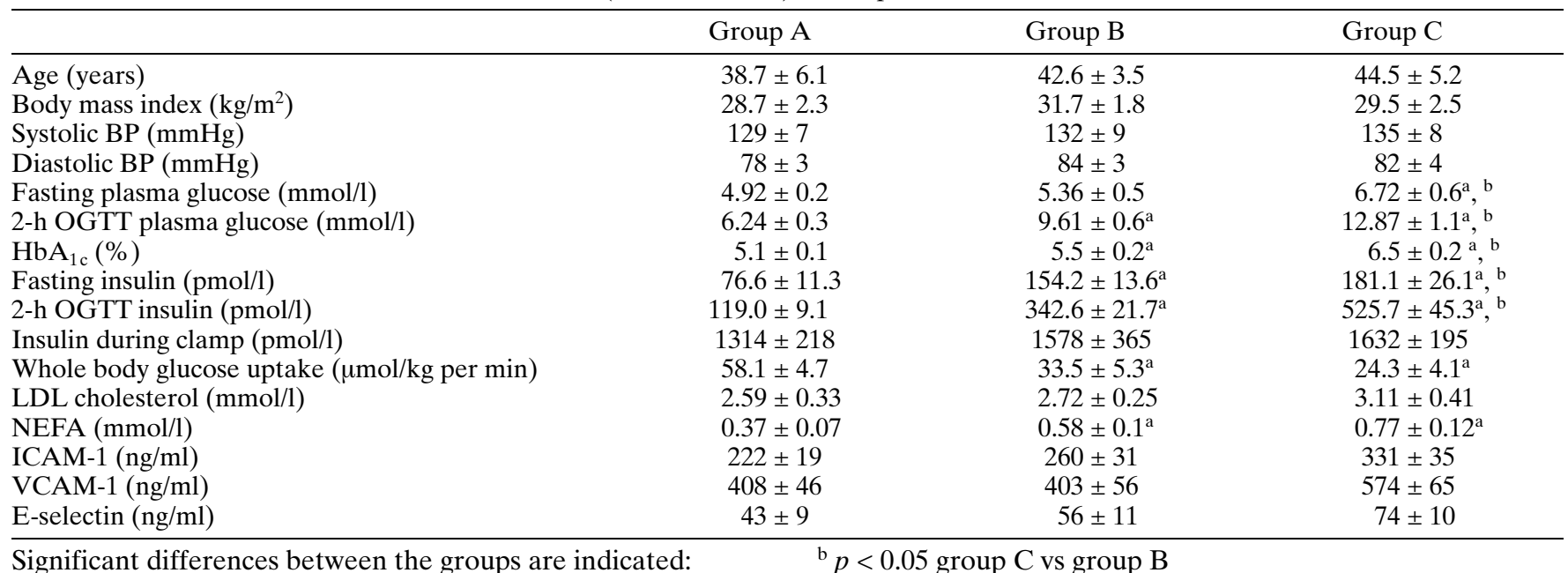

Significant differences between the groups are indicated:

${ }^{\mathrm{a}} p<0.05$ vs healthy control subjects (group A)

used for the frequent sampling. After a priming dose of 1.2 $\mathrm{nmol} / \mathrm{m}^{2}$ insulin the infusion with insulin (actrapid $100 \mathrm{U} / \mathrm{ml}$, Novo Nordisk, Bagsvaerd, Denmark) was started with a constant infusion rate of $0.28 \mathrm{nmol} / \mathrm{m}^{2}$ body surface per min and continued for $120 \mathrm{~min}$. After $3 \mathrm{~min}$ the variable of $20 \%$ glucose infusion rate was added and adjusted during the clamp to maintain the blood glucose at $5.0 \mathrm{mmol} / \mathrm{l}$. Bedside blood glucose measurements were carried out every 5 min using the glucose dehydrogenase technique with haemocue B (haemocue, Angelholm, Sweden). Additional blood samples were taken after $60 \mathrm{~min}$ and during the steady state for the measurement of glucose, insulin, C-peptide, ICAM-1, VCAM-1, and E-selectin.

Assays and calculations. Body mass index was calculated as weight divided by squared height. Waist and hip circumferences were measured. Basal, fasting blood samples were taken after an overnight fast and after a $30 \mathrm{~min}$ rest in the supine position to determine serum lipids, NEFA, leptin, ICAM-1, VCAM-1, E-selectin and standard laboratory parameters. Plasma insulin was measured in a two-site chemiluminescent enzyme immunometric assay for the IMMULITE automated analyser (Diagnostic Products, Los Angeles, Calif., USA). Plasma C-peptide was measured in a solid phase, chemiluminescent enzyme immunoassay using the IMMULITE automated analyser (Diagnostic Products). For the quantification of serum NEFA an in vitro enzymatic colorimetric method was used (NEFA C, ACS-ACOD method, Wako Chemikals, Neuss, Germany). Leptin was measured as described [20].

Serum concentrations of ICAM-1, VCAM-1, and E-selectin were measured by the use of monoclonal antibody-based ELISA assays ( $\mathrm{R}$ and D Systems, Abingdon, UK). All samples were tested in duplicate. Both interassay and intraassay coefficients of variation were less than $5 \%$.

Statistical analysis. Data are shown as means \pm SEM unless otherwise stated. The calculation of insulin sensitivity in the steady state during the second hour of the euglycaemic clamp was carried out as described [18]. All calculations and statistics were done with SPSS for Windows (SPSS, Chicago, Ill., USA). The differences between the groups were tested by one way ANOVA. If the $p$ value was less than 0.05 the groups were compared by the appropriate test (students $t$ test for unpaired samples or chi square test). A $p$-value of less than 0.05 was re- garded as significant. Multivariate regression analyses were done and correlations between variables were tested with Spearman's correlation test. A correlation coefficient ( $r$ ) of $p$ less than 0.05 was accepted as significant. To minimise type I and type II errors, we calculated a statistical power greater than $80 \%$ with a given significant $p$ value of less than 0.05 for the comparisons between the groups and between basal and steady state of the euglycaemic hyperinsulinaemic clamp. The statistical power was between 60 and $80 \%$ depending on the study group for the correlation analyses.

\section{Results}

Subjects. The clinical and biochemical characteristics of the participants are summarized in Table I. Subjects in the control group (group A) had normal fasting C-peptide $(0.88 \pm 0.04 \mathrm{nmol} / \mathrm{l})$ concentrations and were insulin sensitive, as assessed by the euglycaemic hyperinsulinaemic clamp. The subjects in group B had increased fasting glucose but impaired glucose tolerance, elevated fasting $\mathrm{C}$-peptide concentrations $(1.68 \pm 0.2 \mathrm{nmol} / \mathrm{l})$ and still normal $\mathrm{HbA}_{1 \mathrm{c}}$ values, representing a good glycaemic longterm control. However, these subjects were extremely insulin resistant (Table 1). There was no significant difference in the extent of insulin resistance assessed during the clamp between group B and group C (Table 1). Group $\mathrm{C}$ is further characterized by high fasting C-peptide $(1.86 \pm 0.6 \mathrm{nmol} / \mathrm{l})$ concentrations, increased $\mathrm{HbA}_{1 \mathrm{c}}$ values as a correlate of longterm hyperglycaemia. No significant differences $(p<0.05)$ in concentrations of leptin, total cholesterol, HDL and LDL cholesterol, and triglycerides were found between the three groups. Subjects with IGT (group B) and patients with Type II diabetes (group C) had significantly higher plasma concentrations of NEFA than the control subjects (group A) (Table 1). 
A
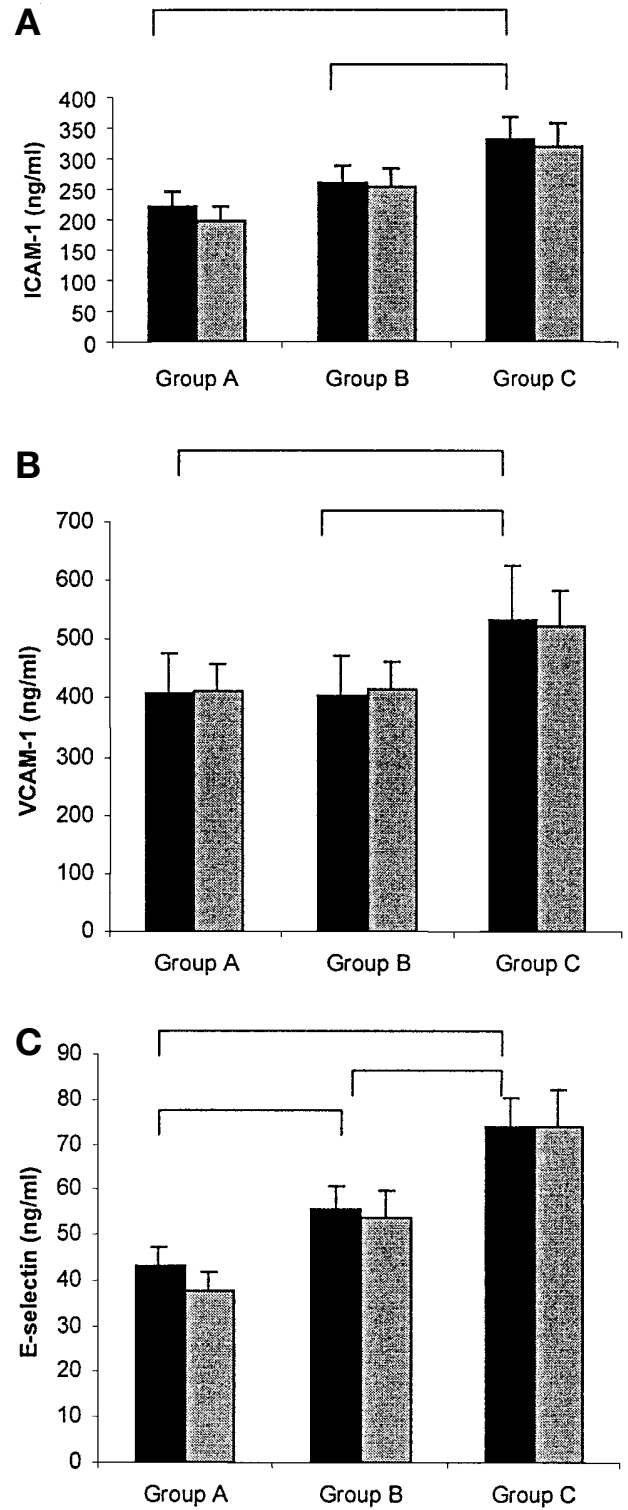

Fig. 1. Plasma soluble ICAM-1 (A), VCAM-1 (B), E-selectin (C) (means \pm SEM) concentrations at baseline (ם) and in the steady state of an euglycaemic hyperinsulinaemic clamp (문) in healthy control subjects (group A, $n=28$ ), patients with impaired glucose tolerance, fasting hyperinsulinaemia, normal fasting glucose and normal $\mathrm{HbA}_{1 \mathrm{c}}$ (group $\mathrm{B}, n=24$ ), patients with diabetes mellitus Type II, long-term hyperglycaemia (increased $\mathrm{HbA}_{1 \mathrm{c}}$ ), and hyperinsulinaemia (group $\mathrm{C}, n=32$ ). Brackets indicate differences between the groups at baseline $(p<0.05)$. There were no significant differences in the soluble adhesion molecule concentrations between baseline and the steady state of the clamp in any group. The calculated statistical power for these group comparisons was $>80 \%$

ICAM-1, VCAM-1, E-selectin plasma concentrations. Patients with Type II diabetes (group C) had significantly higher ICAM-1, VCAM-1, E-selectin plasma concentrations than the control group (group A) and Group B (Fig. 1). In addition, plasma E-selectin concentrations were significantly higher in group B than in group $\mathrm{A}(p<0.05$, Fig. 1$)$. The plasma adhesion molecule concentrations at baseline are shown in Table 1 .

In the analysis of the entire study cohort there were significant correlations $(p<0.05)$ between the plasma ICAM-1, VCAM-1, or E-selectin concentrations and the fasting plasma glucose, the 2-h OGTT plasma glucose, and the $\mathrm{HbA}_{1 \mathrm{c}}$ values (Table 2). These positive correlations between parameters of the glycaemic status and AM concentrations could be confirmed in all three study groups (Table 2). Plasma NEFA concentration correlates significantly with ICAM-1 $\quad(r=0.7, \quad p<0.01), \quad$ VCAM-1 $\quad(r=0.72$, $p<0.001)$ and E-selectin $(r=0.69, p<0.001)$ concentrations in the entire study cohort.

There was also a correlation between plasma E-selectin concentrations and fasting, 2-h OGTT insulin concentrations and the extent of insulin resistance (Table 2) in the entire population. To control for the effect of chronic hyperinsulinaemia and the extent of insulin resistance without chronic hyperglycaemia, group B was compared to the control group (group A) ICAM-1 and VCAM-1 concentrations were indistinguishable between these groups. Despite significant higher fasting and 2-h OGTT insulin concentrations and a significantly lower whole body glucose uptake in the euglycaemic hyperinsulinaemic clamp in group B compared with group A $(p<0.05)$. Although there was a pattern suggesting a trend in increasing concentrations of ICAM-1 across glucose tolerance categories and insulin resistance from normal (group A) to impaired (group B) to diabetic (group C) this trend was not significant $(p<0.05)$ despite the sufficient statistical power $(>80 \%)$.

No correlation could be found between the ICAM-1 and VCAM-1 plasma AM concentrations and fasting or $2 \mathrm{~h}-\mathrm{OGTT}$ insulin concentrations or the extent of insulin resistance in the entire study population (Table 2). Moreover, we were not able to detect correlations $(p<0.05)$ between E-selectin, ICAM-1 and VCAM-1 concentrations and fasting and 2-h OGTT insulin concentrations and the whole body glucose uptake in any group. However, the statistical power for the detection of a significant correlation of these parameters in the different groups was not sufficient. To reach a statistical power greater than $80 \%$, more than 1500 subjects would have been needed. The acute hyperinsulinaemia during the euglycaemic hyperinsulinaemic clamp had no significant effect on the plasma ICAM-1, VCAM-1, or Eselectin concentrations in any group (Fig. 1).

A multiple regression analysis was done to evaluate the potential influence of cardiovascular risk factors (age, sex, BMI, LDL-cholesterol $>3.9 \mathrm{mmol} / \mathrm{l}$, systolic and diastolic blood pressure $>160$ / $95 \mathrm{mmHg}$, smoking) and glucose and insulin concentrations, whole body glucose uptake, $\mathrm{HbA}_{1 \mathrm{c}}$ values and the NEFA concentration on the plasma AM concentrations. This analysis confirmed that fasting and 
Table 2. Correlations between parameters of the glycaemic status, and insulin resistance in the entire study cohort and the plasma concentration of the soluble adhesion molecules

\begin{tabular}{|c|c|c|c|}
\hline Parameter & ICAM-1 & VCAM-1 & E-selectin \\
\hline $\begin{array}{l}\text { Fasting glucose } \\
\text { Group A } \\
\text { Group B } \\
\text { Group C }\end{array}$ & $\begin{array}{l}r=0.62(p<0.001) \\
r=0.55(p<0.05) \\
r=0.68(p<0.05) \\
r=0.61(p<0.05)\end{array}$ & $\begin{array}{l}r=0.56(p<0.01) \\
r=0.50(p<0.05) \\
r=0.61(p<0.05) \\
r=0.62(p<0.05)\end{array}$ & $\begin{array}{l}r=0.59(p<0.01) \\
r=0.51(p<0.05) \\
r=0.63(p<0.05) \\
r=0.63(p<0.05)\end{array}$ \\
\hline $\begin{array}{l}\text { 2h-OGTT glucose } \\
\text { Group A } \\
\text { Group B } \\
\text { Group C }\end{array}$ & $\begin{array}{l}r=0.72(p<0.05) \\
r=0.59(p<0.05) \\
r=0.77(p<0.05) \\
r=0.73(p<0.05)\end{array}$ & $\begin{array}{l}r=0.68(p<0.01) \\
r=0.62(p<0.05) \\
r=0.70(p<0.05) \\
r=0.73(p<0.05)\end{array}$ & $\begin{array}{l}r=0.66(p<0.001) \\
r=0.58(p<0.05) \\
r=0.68(p<0.05) \\
r=0.69(p<0.05)\end{array}$ \\
\hline Fasting insulin concentrations & $r=0.42(p>0.05)$ & $r=0.39(p>0.05)$ & $r=0.62(p<0.05)$ \\
\hline 2-h OGTT insulin concentrations & $r=0.31(p>0.05)$ & $r=0.31(p>0.05)$ & $r=0.65(p<0.05)$ \\
\hline
\end{tabular}

2-h OGTT plasma glucose, the $\mathrm{HbA}_{1 \mathrm{c}}$ values and the NEFA concentration are related to increased AM concentrations $(p<0.05)$. Hyperinsulinaemia and decreased whole body glucose uptake are independent risk factors for increased E-selectin concentrations but not for increased ICAM-1 and VCAM-1 concentrations.

\section{Discussion}

High glucose concentrations in patients with diabetes contribute to increased plasma concentrations of soluble adhesion molecules and could therefore explain in part the increased risk of patients with diabetes mellitus for the development of premature atherosclerosis $[1,2,7]$. There are contradictory reports regarding the effects of hyperglycaemia $[5,7,8]$ or hyperinsulinaemia [13-15] on soluble adhesion molecule concentrations. We therefore investigated if increased ICAM-1, VCAM-1, or E-selectin concentrations are primarily associated with hyperglycaemia or hyperinsulinaemia. Soluble AM concentrations were investigated in patients with hyperinsulinaemia but without chronic hyperglycaemia (group B), patients with combined chronic hyperinsulinaemia and hyperglycaemia (group C) and the control subjects (group A). Although we did not measure the time between the onset of Type II diabetes and our diagnosis of Type II diabetes at the time of OGTT in group C, we can assume that these patients had chronic hyperglycaemia, since their $\mathrm{HbA}_{1 \mathrm{c}}$ values were higher compared with the IGT group (group B). Other risk factors for atherosclerosis, which increase soluble AM concentrations, like hypertension [21], hyperlipidaemia [22], or preexisting cardiovascular disease [10] which could have modified the results of previous studies [23] were not present in our study groups.
However, though none of the patients had clinical evidence for cardiovascular disease we cannot exclude that some of the IGT and diabetic patients had subclinical cardiovascular disease [2]. Therefore, we confirmed by multiple regression analysis that hypertension, increased LDL-cholesterol concentrations, smoking, BMI, age and sex were not related to alterations in the plasma AM concentrations in our study cohort.

Patients with combined chronic hyperglycaemia and hyperinsulinaemia (group C) had higher plasma AM concentrations than the patients with hyperinsulinaemia but without chronic hyperglycaemia (group B) suggesting that chronic hyperglycaemia is the primary cause for increased AM concentrations in diabetic patients. As further evidence for the primary relation between glucose concentrations and plasma AM concentrations, we found a correlation between ICAM-1, VCAM-1, and E-selectin plasma concentrations and parameters of glycaemic control: fasting and 2-h OGTT plasma glucose and $\mathrm{HbA}_{1 \mathrm{c}}$ value (Table 2) independently from the glycaemic status in all groups. Our results are in accordance with hyperglycaemic clamp studies in healthy subjects which show that hyperglycaemia causes an increase in plasma AM concentrations [7]. Moreover, an association of E-selectin concentrations with $\mathrm{HbA}_{1 \mathrm{c}}$ values in patients with Type I diabetes $[4,5]$ and with Type II diabetes [8] has been found. However, other recent studies could not detect a relation between AM concentrations and glycaemic control $[9,24]$. These conflicting results could be explained by differences in the degree of glycaemic control between these different study cohorts $[4,24]$. The influence of the glycaemic control on plasma AM concentrations is further suggested by results showing that the association between $\mathrm{HbA}_{1 \mathrm{c}}$ and E-selectin disappeared after improvement of glycaemic control [8]. 
The role of hyperinsulinaemia in the modulation of circulating AM concentrations as a primary cause of increased AM concentrations in patients with Type II diabetes is controversial. A correlation was found between increased 2-h OGTT insulin concentrations and increased ICAM-1 plasma concentrations in essential hypertensive patients with IGT and hyperlipidaemia [14]. We did not find an association between increased fasting or 2-h OGTT insulin concentrations and increased ICAM-1 and VCAM-1 concentrations in any group. The absence of the confounding factors on AM concentrations, hypertension and hyperlipidaemia, in our study cohort which is in contrast with the subjects from another study [14] could explain these contradictory results.

Chronic hyperinsulinaemia was only associated with increased E-selectin concentrations but not with increased ICAM-1 or VCAM-1 plasma concentrations. This suggests that plasma concentrations of different adhesion molecules are differentially associated with long-term hyperinsulinaemia or that the observed relation between hyperinsulinaemia and E-selectin concentrations represents a secondary phenomenon. We obtained further evidence that hyperinsulinaemia is not likely to be the primary cause for increased AM concentrations from euglycaemic hyperinsulinaemic clamp studies. Hyperinsulinaemia during the euglycaemic hyperinsulinaemic clamp did not cause increased plasma AM concentrations in any group. In accordance with our result, another study [15] showed that plasma concentrations of adhesion molecules were not affected by 30 -fold increased insulin concentrations during a 6-h euglycaemic hyperinsulinaemic clamp [15]. However, in this study only healthy subjects without chronic hyperinsulinaemia were investigated and even the 6-h euglycaemic hyperinsulinaemic clamps might not reflect the effects of long-term hyperinsulinaemia or the cardiovascular susceptibility of patients at an early stage of the development of diabetes (group B).

A study [13] showed that the concentrations of soluble ICAM-1, VCAM-1, and E-selectin correlate with the degree of insulin resistance calculated from a modified insulin suppression test [25] in healthy volunteers. We did not find a correlation between the extent of insulin resistance, determined by the euglycaemic hyperinsulinaemic clamp and the concentration of plasma ICAM-1 or VCAM-1 in any group. However, there was a correlation between whole body glucose uptake and E-selectin concentrations. This result further suggests that different groups of AM, such as the selectin family (E-selectin) and the immunoglobulin supergene family (ICAM-1, VCAM-1) are differentially associated with hyperinsulinaemia and insulin resistance.

In conclusion, our data suggest that increased ICAM-1 and VCAM-1 plasma concentrations are primarily related to chronic hyperglycaemia rather than to hyperinsulinaemia. E-selectin plasma concentrations but not ICAM-1 and VCAM-1 concentrations, are also related to hyperinsulinaemia and the degree of insulin resistance, suggesting that different groups of AM are differentially associated to insulin concentrations and insulin resistance.

Acknowledgements. This paper was supported by the Bundesministerium für Bildung und Forschung $(\mathrm{BMB}+\mathrm{F})$, Interdisciplinary Centre for Clinical Research (IZKF) at the University of Leipzig (project B 15)

\section{References}

1. Colwell JA (1993) Vascular thrombosis in type II diabetes mellitus. Diabetes 42: 8-11

2. Haffner SM, Lehto S, Ronnemaa T, Pyorala K, Laakso M (1998) Mortality from coronary heart disease in subjects with type 2 diabetes and in nondiabetic subjects with and without prior myocardial infarction. N Engl J Med 339: 229-234

3. Gearing AJH, Hemingway I, Pigott R, Hughes J, Rees AJ, Cashman SJ (1992) Soluble forms of vascular adhesion molecules, E-selectin, ICAM-1, and VCAM-1: pathological significance. Ann NY Acad Sci 667: 324-331

4. Fasching P, Veitl M, Rohac M et al. (1996) Elevated concentrations of circulating adhesion molecules and their association with microvascular complications in insulin-dependent diabetes mellitus. J Clin Endocrinol Metab 81: 4313-4317

5. Cominacini L, Fratta Pasini A, Garbin U et al. (1995) Elevated levels of soluble E-selectin in patients with IDDM and NIDDM: relation to metabolic control. Diabetologia 38: $1122-1124$

6. Otsuki M, Hashimoto K, Morimoto Y, Kishimoto T, Kasayama S (1997) Circulating vascular cell adhesion molecule-1 (VCAM-1) in atherosclerotic NIDDM patients. Diabetes 46: 2096-2101

7. Marfella R, Esposito K, Giunta R et al. (2000) Circulating adhesion molecules in humans. Role of hyperglycemia and hyperinsulinemia. Circulation 101: 2247-2251

8. Cominacini L, Fratta Pasini A, Garbin U et al. (1997) E-selectin plasma concentration is influenced by glycemic control in NIDDM patients: possible role of oxidative stress. Diabetologia 40: 584-589

9. Steiner M, Reinhardt KM, Krammer B, Ernst B, Blann AD (1994) Increased levels of soluble adhesion molecules in type 2 (non-insulin dependent) diabetes mellitus are independent of glycaemic control. Thromb Haemost 72: 979-984

10. Ridker PM, Hennekens CH, Roitman-Johnson B, Stampfer MJ, Allen J (1998) Plasma concentration of soluble intercellular adhesion molecule-1 and risk of future myocardial infarction in apparently healthy men. Lancet 531: 88-92

11. Lim SC, Caballero AE, Smakowski P, LoGerfo FW, Horton ES, Veves A (1999) Soluble Intercellular adhesion molecule, vascular cell adhesion molecule, and impaired microvascular reactivity are early markers of vasculopathy in type 2 diabetic individuals without microalbuminuria. Diabetes Care 22: 1865-1870

12. Blann AD, Lip GYH (1998) Endothelial integrity, soluble adhesion molecules and platelet markers in type 1 diabetes mellitus. Diabet Med 15: 634-642 
13. Chen NG, Holmes M, Reaven GM (1999) Relationship between insulin resistance, soluble adhesion molecules, and mononuclear cell binding in healthy volunteers. J Clin Endocrinol Metab 84: 3485-3489

14. Ferri C, Desideri G, Baldoncini R et al. (1998) Early activation of vascular endothelium in nonobese, nondiabetic essential hypertensive patients with multiple metabolic abnormalities. Diabetes 47: 660-667

15. Jilma B, Dallinger S, Hergovich N, Eichler HG, Richter V, Wagner OF (2000) Effects of hyperinsulinemia on plasma levels of circulating adhesion molecules. J Clin Endocrinol Metab 85: 1748-1751

16. The Expert Committee on the Diagnosis and Classification of Diabetes mellitus (1998): Report the Expert Committee on the Diagnosis and Classification of Diabetes mellitus (Committee Report). Diabetes Care 21 [Suppl 1]: S5-S19

17. Ferrannini E, Natali A, Bell P, Cavallo-Perin P, Lalic N, Mingrone G (1997) Insulin resistance and hypersecretion in obesity. J Clin Invest 100: 1166-1173

18. DeFronzo RA, Tobin JD, Andres R (1979) Glucose clamp technique: a method for quantifying insulin secretion and resistance. Am J Physiol 237: E214-E223

19. Vauhkonen I, Niskanen L, Vanninen E, Kainulainen S, Uusitupa M, Laakso M (1997) Defects in insulin secretion and insulin action in non-insulin-dependent diabetes mellitus are inherited. J Clin Invest 100: 86-96
20. Blüher M, Kratzsch J, Paschke R (2001) Plasma levels of tumor necrosis factor- $\alpha$, angiotensin II, growth hormone and IGF-1 are not elevated in insulin resistant obese individuals with impaired glucose tolerance. Diabetes Care 24: 328-334

21. Blann AD, Tse W, Maxwell SRJ, Waite MA (1994) Increased levels of soluble adhesion molecule E-selectin in essential hypertension. J Hypertens 112: 925-928

22. Blann AD, Seigneur M, Steiner M, Niler JP, McCollum CN (1998) Circulating ICAM-1 and VCAM-1 in perpheral artery disease and hypercholesterolemia: relationship to the location of atherosclerotic disease, smoking, and in the prediction of adverse events. Thromb Haemost 79: 1080-1085

23. Blann AD, Lip GYH (2000) Editorial: Cell adhesion molecules in cardiovascular disease and its risk factors - what can soluble levels tell us? J Clin Endocrinol Metab 85: 1745-1747

24. Bannan S, Mansfield MW, Grant PJ (1998) Soluble vascular cell adhesion molecule-1 and E-selectin levels in relation to vascular risk factors and to E-selectin genotype in the first degree relatives of NIDDM patients and in NIDDM patients. Diabetologia 41: 460-466

25. Harano Y, Ohgaku S, Hidaka H et al. (1977) Glucose, insulin and somatostatin infusion for the determination of insulin sensitivity. J Clin Endocrinol Metab 45: 1124-1127 